Twisting thread

Science 343, 868-872 (2014)

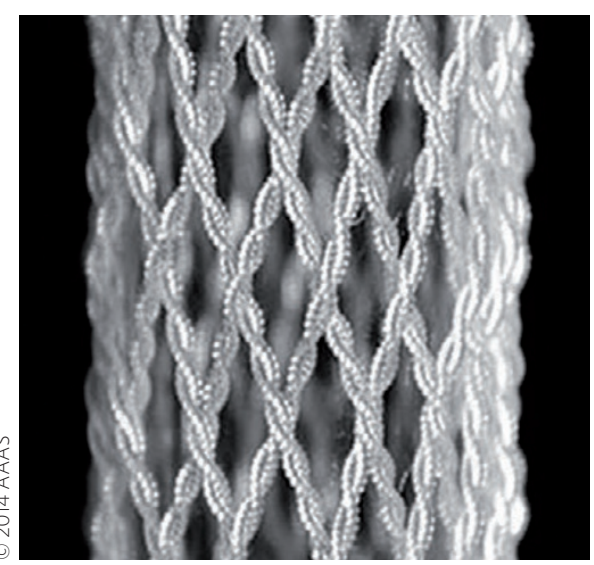

Artificial muscles - most often actuating fibres that could be used in prosthetic limbs, temperature-adjusting textiles and microfluidic pumps - have been made of a variety of materials, such as conducting organic polymers, shape-memory polymers or metals, or polymer/carbon-nanotube composites. However, these suffer from short cycle life, low work capacity, hysteresis or high cost. Now, Ray Baughman and colleagues show that when polyethylene or nylon fibres - of the kind used in sewing thread or fishing line - are appropriately twisted to a high degree, they make coiled muscles that contract to half their length when heated, generating power at a density comparable to that of a jet engine. The researchers explain that the high performance of these fibres results from the untwisting of the fibres as a result of both length contraction and fibrediameter expansion during heating. They also show that the fibres retain their performance after over a million actuation cycles, and that the muscles can be made to expand on heating by coiling the twisted fibres with opposite chirality to that of the twisting.

\section{Intermolecular intrusions \\ Nature Commun. 5, 3321 (2014)}

Peptides that can disrupt or permeate cell membranes have been exploited in various biomedical applications including gene delivery and as antimicrobials. The interactions between cationic and hydrophobic regions of peptides and cell membranes are well established. Now, Samuel Stupp and colleagues investigate the influence of intermolecular interactions within peptide amphiphiles (PAs) on cell viability and membrane integrity. PAs comprise a peptide chain attached at one end to an alkyl chain and self-assemble into nanofibrous structures. Stupp and colleagues alter the degree of intermolecular hydrogen bonding within the PAs by changing the amino acid sequence whilst keeping the cationic and hydrophobic regions as similar as possible. Coatings formed from PAs with a lower tendency to form $\beta$-sheet hydrogen-bonded structures (and, as a result, have reduced intermolecular cohesion) induce cell death in a range of cell lines, however, coatings formed from PAs with increased hydrogenbonding capability support cell viability. In cell-membrane model studies using liposomes, the PA assemblies with reduced intermolecular cohesion are shown to interact with and disrupt the lipid domains of a phospholipid bilayer. AS

\section{Acoustic diodes}

Nature Commun. 5, 3398 (2014)

The development of optical metamaterials has brought, as a consequence, the birth of a parallel field in acoustics, which concentrates on the design and realization of devices that can mimic their exotic properties with mechanical waves. One important device to be implemented is the acoustic diode: an instrument capable of transmitting acoustic energy in one direction and no energy in the opposite direction. Most acoustic diodes

\title{
Rapid current switching
}

To reduce the static power consumption of electronic circuits, the current flowing in a transistor should ideally switch from an on to off state by applying minimum variations to the gate voltage. In traditional Si-based electronics, thermodynamics limits the maximum rate of this transition, whereas this is not the case for nanoelectromechanical switches - where current is controlled by mechanical nanoactuators. Ji-Hun Kim and colleagues now realize a three-terminal transistor by suspending a Ge-Si nanowire above a gate electrode. When the voltage applied to the gate exceeds a threshold value, the nanowire is pulled down by electrostatic attraction and the electric current flowing into it drastically decreases. This transition is reversible and its rate is up to ten times higher than that achieved in traditional transistors. The maximum operating frequency, determined by the flexural resonant frequency of the suspended nanowire, is higher than $100 \mathrm{MHz}$, exceeding that of previously demonstrated microelectromechanical switches. Further improvements in the performance are expected by using channels based on graphene or carbon nanotubes.

proposed so far involve the use of phononic crystal filters. These devices need to be larger than the wavelength they filter, which limits their use for audio and low-frequency applications, in the wavelength range of a few metres and above. Bogdan-Ioan Popa and Steven Cummer now propose a novel pathway to overcome this limitation: the use of a subwavelength acoustic metamaterial. Using Helmholtz cavities coupled with a piezoelectric membrane they realize a tunable broadband acoustic device (with dimensions of a few centimetres) that can be switched between a forward, energy-transmitting configuration and a reverse, non-transmitting one, even at low frequencies and low sound levels.

\section{Live diffraction}

Phys. Rev. Lett. 112, 088102 (2014)

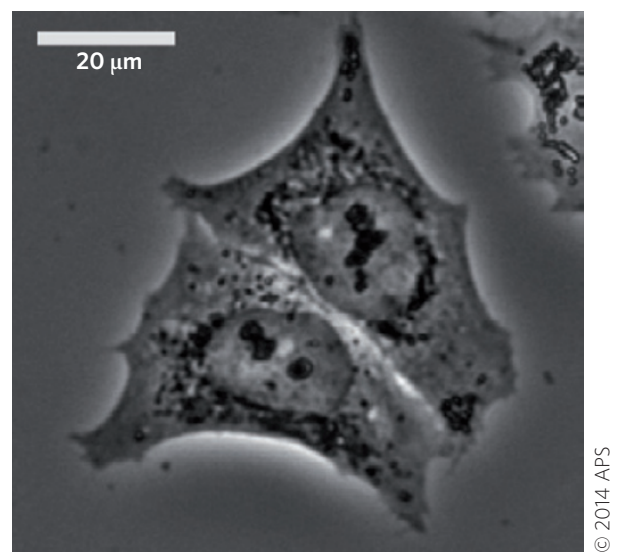

Diffraction techniques based on X-rays are routinely used to determine the structure and composition of materials ranging from inorganic minerals to biological specimens. Recent technical developments have made very small beam sizes possible, which, in turn, allows for small samples to be probed, well below the size of biological cells. However, analysing the structure of actual living cells remains a challenge due to their weak contrast. One way of enhancing this is to investigate the cells in dried form, but this comes at the cost of observing them in their natural state. To overcome this limitation, Britta Weinhausen and colleagues have developed a microfluidic device that mimics their natural environment, and is transparent to X-rays. By passing the cells through an X-ray beam that is only a few hundred nanometres wide, they are able to image them while they are alive, and show that chemically fixed cells tend to display artefacts. Scientists looking to perform X-ray imaging studies of biological material in the future should take note.

Written by Luigi Martiradonna, Olivia Nicoletti, Pep Pàmies, Alison Stoddart and Andrea Taroni. 